\title{
Der Streifen-Meister
}

LUKAS WALLA Das kinesiologische Taping hat unter den Physiotherapeuten nicht

nur Freunde - bis heute ist dessen Wirksamkeit nicht wissenschaftlich belegt. Immer wieder beschäftigen sich Therapeuten mit diesem Thema. So auch Lukas Walla, der in seiner Bachelorarbeit die kurzfristige Wirkung kinesiologischen Tapings untersuchte.

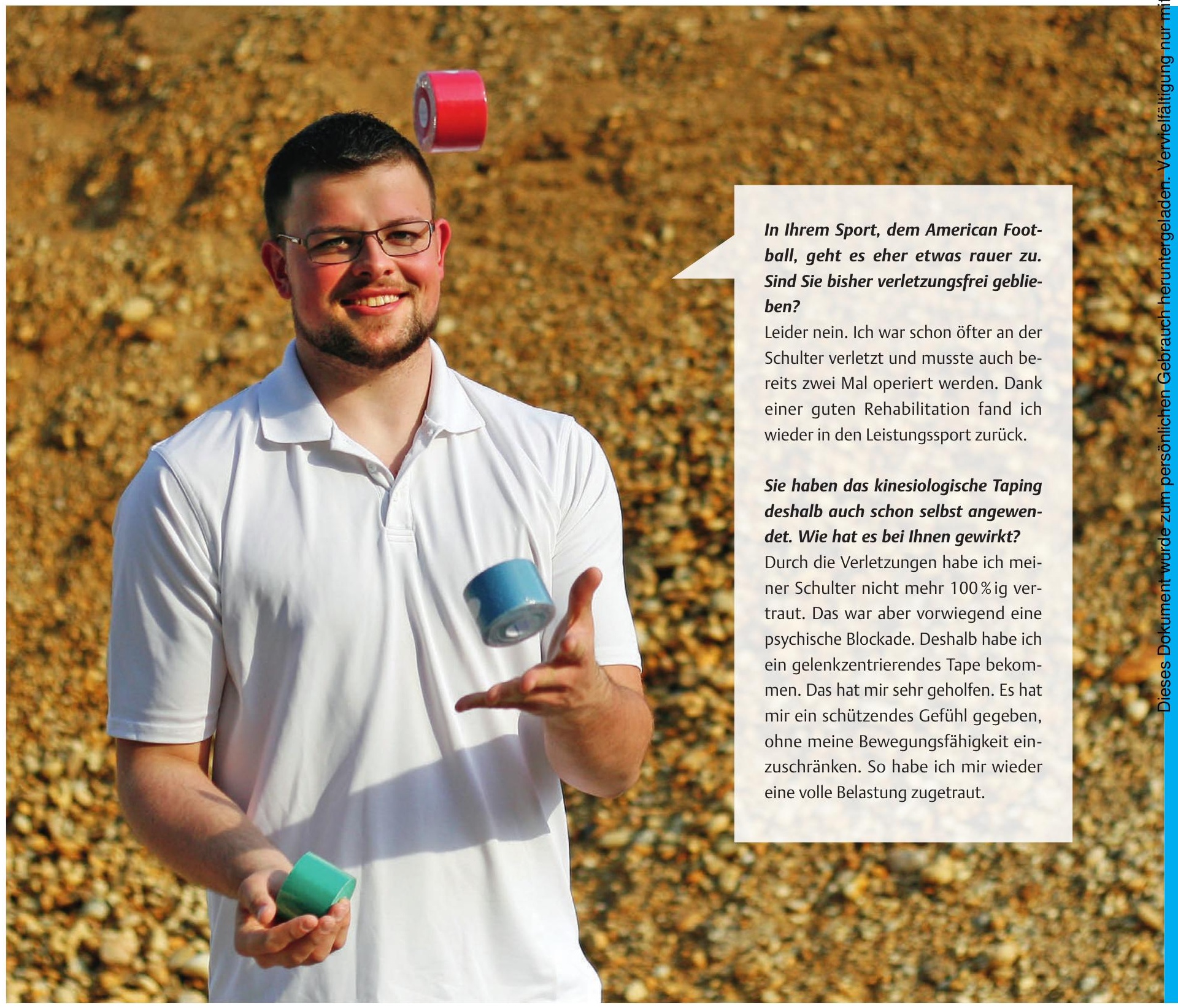




\section{Kinesiologisches Taping bei unspezifischen Nackenschmerzen}

\section{Die Bachelorarbeit}

Kinesiologisches Taping ist in der Physiotherapie mittlerweile sehr verbreitet und wird bei zahlreichen muskuloskeletalen und neurologischen Krankheitsbildern eingesetzt. Hinter dem Begriff verbergen sich verschiedene Methoden, wie zum Beispiel das Kinesio-Taping, das K-Taping oder das Medi-Taping. Sie arbeiten mit unterschiedlichen Aspekten und Anlagetechniken, wie einer Farblehre oder dem Hautvorschub. Ihnen allen gemein ist, dass ihre Wirkweise und Effektivität in der Therapie bisher wissenschaftlich nicht nachgewiesen sind. Vielmehr beruhen die aktuellen Erkenntnisse zum kinesiologischen Taping vor allem auf Anwendungserfahrungen. Lukas Walla hat deshalb beschlossen, die kurzfristigen Auswirkungen der Klebestreifen in seiner Bachelorarbeit am Beispiel des unspezifischen Nackenschmerzes empirisch zu untersuchen. Diese Schmerzen haben oftmals myofasziale Ursachen und gehen mit einer Druckempfindlichkeitssteigerung des Weichteilgewebes einher. Als Design für seine Arbeit wählte er eine einfach verblindete, prospektive, randomisierte Fall-Kontroll-Studie und bezog darin 21 Frauen und zwei Männer im Alter zwischen 19 und 47 Jahren ein. Diese verteilte er zufällig in eine Interventions- und eine Kontrollgruppe. Bei allen Probanden maß er an sechs Punkten im Schulter-Nacken-Bereich jeweils die Druckschmerzgrenze und die Druckschmerztoleranz mithilfe eines Druckkraftmessgeräts (Newtonmeter). Die Interventionsgruppe erhielt im Anschluss für den M. trapezius descendens und den $\mathrm{M}$. levator scapulae eine muskeldetonisierende Tape-Anlage nach dem K-Taping Academy International Konzept. Die Kontrollgruppe behandelte Lukas Walla mit dem optisch gleichen K-Taping, verzichtete aber bei der Anlage auf die therapeutischen Vorgaben. Diese Art der Anlage wird als Shamtape (Scheintape) bezeichnet. Zudem erhielten alle Probanden ein Heimprogramm mit fünf Übungen - eine Kräftigungsübung des Schultergürtels, zwei Übungen für die Mobilisation der Halswirbelsäule und eine Dehnübung für den Schultergürtel, die auf beiden Seiten durchzuführen war. 72 Stunden später wiederholte er die Messungen.

\section{Ergebnisse}

Lukas Walla hat herausgefunden, dass ...

> die Druckschmerztoleranz am Messpunkt über dem linken $M$. levator scapulae in der Interventionsgruppe signifikant größer war $(p=0,04)$ als in der Kontrollgruppe. Die Messungen an allen anderen Punkten ergaben keinen signifikanten Unterschied weder bei der Druckschmerzgrenze, noch bei der Druckschmerztoleranz.

> daher bei der Druckschmerztoleranz die konzeptgeklebte Anlage effektiver zu sein scheint als die konzeptfrei geklebte.

> sich in Bezug auf die Druckschmerzgrenze die Effekte des Shamtapes und die der therapeutischen Tape-Anlage einander sehr ähneln.

\section{Fazit}

Zusammenfassend kann Lukas Walla festhalten, dass ...

> die Behandlung mit kinesiologischem Tape vermutlich zumindest teilweise von psychologischen Faktoren abhängt.

> er nicht nachweisen konnte, dass eine konzeptbasierte Tape-Anlage bei unspezifischen Nackenschmerzen eine höhere schmerzlindernde Wirkung hat, da die konzeptbasierte Anlage bei der Druckschmerztoleranz nur an einem von sechs Punkten signifikante Veränderungen bewirkte und sich bei der Druckschmerzgrenze gar keine signifikanten Änderungen zeigten. Der schmerzreduzierende Effekt kann also eher dem Tape an sich zugeschrieben werden als der Anlagetechnik.

$>$ weitere Studien, auch an anderen Gelenken, erforderlich sind, um mehr über Wirkmechanismen und Effekte des kinesiologischen Tapings als Therapieunterstützung bei muskuloskeletalen Problemen zu erfahren und dessen Langzeitwirkung. Eva Trompetter

$\rightarrow$ Walla, L. Auswirkungen unterschiedlicher Anlagetechniken von Kinesiotape auf Schmerzschwelle und Schmerztoleranz am Beispiel des nichtspezifischen Nackenschmerzes. Bachelorarbeit an der Fachhochschule Campus Wien; 2013 ders interessieren ihn dabei Therader direkten physiotherapeutischen Behandlung weiterwirken, wie das kinesiologische Taping. 